\title{
Refined Restricted Permutations
}

\author{
Aaron Robertson ${ }^{1}$, Dan Saracino ${ }^{1}$, and Doron Zeilberger ${ }^{2 *}$ \\ ${ }^{1}$ Department of Mathematics, Colgate University, Hamilton, NY 13346, USA \\ aaron@math.colgate.edu, dsaracino@mail.colgate.edu \\ ${ }^{2}$ Department of Mathematics, Rutgers University, New Brunswick, NJ 08903, USA \\ zeilberg@math.rutgers.edu
}

Received October 28, 2002

AMS Subject Classification: 05A15, 68R15

Dedication: In memory of Rodica Simion (1955-2000)

This article is dedicated to the memory of Rodica Simion, one of the greatest enumerators of the $20^{\text {th }}$ century. Both derangements [8] and restricted permutations [10] were very dear to her heart, and we are sure that she would have appreciated the present surprising connections between these at-first-sight unrelated concepts.

Abstract. Define $S_{n}^{k}(\alpha)$ to be the set of permutations of $\{1,2, \ldots, n\}$ with exactly $k$ fixed points which avoid the pattern $\alpha \in S_{m}$. Let $s_{n}^{k}(\alpha)$ be the size of $S_{n}^{k}(\alpha)$. We investigate $S_{n}^{0}(\alpha)$ for all $\alpha \in S_{3}$ as well as show that $s_{n}^{k}(132)=s_{n}^{k}(213)=s_{n}^{k}(321)$ and $s_{n}^{k}(231)=s_{n}^{k}(312)$ for all $0 \leq k \leq n$.

Keywords: restricted permutations, Fine's sequence, Dyck paths

\section{Introduction}

Let $\pi \in S_{n}$ be a permutation of $\{1,2, \ldots, n\}$ written in one-line notation. Let $\alpha \in S_{m}$. We say that $\pi$ contains the pattern $\alpha$ if there exist indices $i_{1}<i_{2}<\cdots<i_{m}$ such that $\pi_{i_{1}} \pi_{i_{2}} \ldots \pi_{i_{m}}$ is equivalent to $\alpha$, where we define equivalence as follows. Define $\bar{\pi}_{i_{j}}=$ $\left|\left\{x: \pi_{i_{x}} \leq \pi_{i_{j}}, 1 \leq x \leq m\right\}\right|$. If $\alpha=\bar{\pi}_{i_{1}} \bar{\pi}_{i_{2}} \ldots \bar{\pi}_{i_{m}}$ then we say that $\alpha$ and $\pi_{i_{1}} \pi_{i_{2}} \ldots \pi_{i_{m}}$ are equivalent. For example, if $\tau=124635$ then $\tau$ contains the pattern 132 by noting that $\tau_{2} \tau_{4} \tau_{5}=263$ is equivalent to 132 . We say that $\pi$ is $\alpha$-avoiding if $\pi$ does not contain the pattern $\alpha$. In our above example, $\tau$ is 321 -avoiding.

Define $S_{n}(\alpha), \alpha \in S_{m}$, to be the set of $\alpha$-avoiding permutations in $S_{n}$. Let $s_{n}(\alpha)$ be the size of $S_{n}(\alpha)$. Knuth [3] showed that, regardless of the pattern $\alpha \in S_{3}, s_{n}(\alpha)=C_{n}=$ $\frac{1}{n+1}\left(\begin{array}{c}2 n \\ n\end{array}\right)$, the $n^{\text {th }}$ Catalan number. Bijective results are given in $[4,5,10,13]$.

We refine the investigation of $S_{n}(\alpha)$ in the following fashion. Let $\alpha \in S_{m}$. Define $S_{n}^{k}(\alpha)$ to be the set of $\alpha$-avoiding permutations of $\{1,2, \ldots, n\}$ with exactly $k$ fixed points. Let $s_{n}^{k}(\alpha)$ be the size of $S_{n}^{k}(\alpha)$. We may write $D_{n}(\alpha)$ and $d_{n}(\alpha)$ for $S_{n}^{0}(\alpha)$ and $s_{n}^{0}(\alpha)$, respectively, since we are dealing with derangements.

${ }^{*}$ Supported in part by the NSF. 


\section{Similarity Relations, Catalan Sequences, and Fine's Sequence}

We begin our investigation with similarity relations. A similarity relation, $R$, is a binary relation on an ordered set which is reflexive, symmetric, but not necessarily transitive, with the condition that if $i R k$ and $i<j<k$ then $i R j$ and $j R k$. Furthermore, we have the following definition about the structure of a similarity relation.

Definition 2.1. A similarity relation, $R$, is said to have $k$ isolated points if $k$ is the number of $i \in\{1,2, \ldots, n\}$ such that there does not exist $j \neq i$ with $i R j$. If $k=0$, we say that the similarity relation is nonsingular. We denote by $S R_{n}(k)$ the set of similarity relations on $\{1,2, \ldots, n\}$ with $k$ isolated points.

There are two common structures which can be used to view similarity relations: graphs and sequences. We will be using the sequence interpretation of a similarity relation as given by Strehl in [12]: Let $R$ be a similarity relation on $\{1,2, \ldots, n\}$. Then $R$ corresponds to an integer sequence $r_{1} r_{2} \ldots r_{n}$ defined for any $1 \leq i \leq n$ by $r_{i}=i-j$, where $j$ is the smallest element of $\{1,2, \ldots, n\}$ such that $i R j$. Throughout this paper we assume that similarity relations are defined on $\{1,2, \ldots, n\}$. To this end, we make the following definition.

Definition 2.2. The set of similarity relations (on $\{1,2, \ldots, n\}$ ) is given by

$$
S R_{n}=\left\{r_{1} r_{2} \cdots r_{n}: r_{i} \in \mathbb{Z}, r_{1}=0 \text { and } 0 \leq r_{i+1} \leq r_{i}+1 \text { for } 1 \leq i \leq n-1\right\} .
$$

For example $S R_{3}=\{000,001,010,011,012\}$.

It is known [7,12] that for $n \geq 1,\left|S R_{n}(0)\right|=F_{n}$, where $F_{n}$ is the $n^{\text {th }}$ Fine number. The first few values of Fine's sequence are $0,1,2,6,18,57,186,622,2120, \ldots$, a sequence first discovered in [2].

Looking at $S R_{n}(0)$ a little more closely, we see that $s \in S R_{n}(0)$ if and only if $s$ has no occurrence of 00 and does not end with 0 . Hence, we say that a nonsingular similarity relation has no double zero, where we consider an ending 0 to be a double zero.

We now state some of the results concerning Fine's sequence as given in $[7,9,12]$.

Theorem 2.1. Let $C_{n}=\frac{1}{n+1}\left(\begin{array}{c}2 n \\ n\end{array}\right)$ and $F_{n}$ be the $n^{\text {th }}$ Catalan number and Fine number, respectively. We have the following for $n \geq 2$ :

1. $\left|S R_{n}(0)\right|=F_{n}$,

2. $C_{n}=2 F_{n}+F_{n-1}$,

3. $F_{n}=\sum_{1 \leq k \leq n / 2}\left(\begin{array}{c}2 n-2 k-1 \\ n-1\end{array}\right)-\left(\begin{array}{c}2 n-2 k-1 \\ n\end{array}\right)$,

4. $F_{n}=\frac{1}{2} \sum_{i=0}^{n-2}\left(\frac{-1}{2}\right)^{i} C_{n-i}$.

As we can see, the Catalan and Fine numbers are related. This becomes more evident in light of the following definition.

Definition 2.3. The set of Catalan sequences of length $n$ is given by

$$
\operatorname{Cat}(n)=\left\{c_{1} c_{2} \cdots c_{n}: c_{i} \in \mathbb{Z}, 1 \leq c_{1} \leq c_{2} \leq \cdots \leq c_{n} \text {, and } c_{i} \leq i \text { for } 1 \leq i \leq n\right\} .
$$

For example: $\operatorname{Cat}(3)=\{111,112,113,122,123\}$. It is well-known and easy to see that the cardinality of $\operatorname{Cat}(n)$ is the $n^{\text {th }}$ Catalan number. It is also well-known and easy to see that the generating function for the Catalan numbers, $\psi(t)=\sum_{n=0}^{\infty} C_{n} t^{n}$, satisfies the quadratic equation $\psi(t)=1+t \psi^{2}(t)$, and hence that $\psi(t)=\frac{1-\sqrt{1-4 t}}{2 t}$. 


\section{321-Avoiding Derangements and Dyck Paths}

The aim of this section is to show that the 321-avoiding derangements are enumerated by Fine's sequence. We will investigate two bijections, the main one due to Krattenthaler [4]. First, we must introduce a few definitions.

Definition 3.1. We call a permutation $\pi \in S_{n}$ a backward derangement if $\pi_{n+1-i} \neq i$ for all $1 \leq i \leq n$.

Consequently, a 123-avoiding backward derangement when read from right to left is a 321-avoiding derangement.

We will use a bijection due to Krattenthaler [4] from $S_{n}(123)$ to the set of Dyck paths of length $2 n$, so for completeness we define a Dyck path.

Definition 3.2. A Dyck path is a path in $\mathbb{R}^{2}$ from $(0,0)$ to $(2 n, 0)$ consisting of a sequence of steps of length $\sqrt{2}$ and slope \pm 1 which does not fall below the $x$-axis. We denote these two types of steps by $(1,1)$ and $(1,-1)$, called up-steps and down-steps, respectively. We say that the length of such a Dyck path is $2 n$ (its horizontal length) and denote the set of Dyck paths of length $2 n$ by Dyck $(2 n)$.

We also have the following definition about certain Dyck paths.

Definition 3.3. We say that a Dyck path contains a hill if it has a peak at height 1 . We say that a Dyck path is hill-free is it contains no hill. We denote the set of hill-free Dyck paths of length $2 n$ by Dyck ${ }^{h f}(2 n)$.

We now describe a natural bijection from $S R_{n}$ to Dyck paths of length $2 n$. Let $s=s_{1} s_{2} \ldots s_{n} \in S R_{n}$. This bijection is very similar to the one given by Krattenthaler [4] from $S_{n}(132)$ to $\operatorname{Dyck}(2 n)$.

Each $s_{i}, 1 \leq i \leq n$, corresponds to the starting height of an up-step. Proceeding from $s_{i}$ to $s_{i+1}$, if $s_{i+1}>s_{i}$ then we continue with up-steps. If $s_{i+1} \leq s_{i}$ we append $s_{i}-s_{i+1}+1$ down-steps followed by a single up-step. This assures us that $s_{i+1}$ corresponds to a starting height of $s_{i+1}$ for an up-step. After $s_{n}$ we use as many down-steps as necessary to end at $(2 n, 0)$. The inverse bijection is obvious. An example is in order.

Let $s=0120121 \in S R_{7}(0)$. This nonsingular similarity relation corresponds to the following Dyck path, with the entries of $s$ marked on the Dyck path.

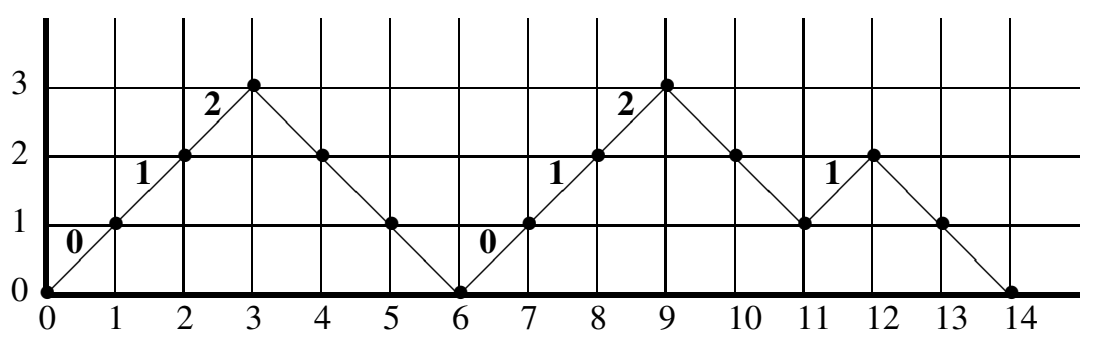

Figure 1: Dyck path corresponding to $0120121 \in S R_{7}(0)$ and to $6573142 \in S_{7}(123)$. 
Using the above bijection and the fact that $|\operatorname{Dyck}(2 n)|=C_{n}$, we easily obtain $\left|S R_{n}\right|=$ $C_{n}$ (which was shown in [7] and [12]). Furthermore, we get the following theorem $\left(\operatorname{SR}_{n}(0)=F_{n}\right.$ was shown in [7] and [12] while $\left|\operatorname{Dyck}^{h f}(2 n)\right|=F_{n}$ was shown in [1]).

Theorem 3.1. For $n \geq 1,\left|S R_{n}(0)\right|=\left|\operatorname{Dyck}^{\text {hf }}(2 n)\right|=F_{n}$, where $F_{n}$ is the $n^{\text {th }}$ Fine number

Proof. Clearly, a hill occurs in a Dyck path if and only if the corresponding similarity relation contains a double zero.

We are now ready to use Krattenthaler's bijection [4], described as follows. Let $\pi=\pi_{1} \pi_{2} \cdots \pi_{n} \in S_{n}(123)$. Determine the right-to-left maxima of $\pi$, i.e., $m=\pi_{i}$ is a right-to-left maximum if $m>\pi_{j}$ for all $j>i$. For example, the right-to-left maxima of 6573142 are in bold type.

Let $\pi$ have right-to-left maxima $m_{1}<m_{2}<\cdots<m_{s}$, so that we may write

$$
\pi=w_{s} m_{s} w_{s-1} m_{s-1} \cdots w_{1} m_{1}
$$

So, for example, in 6573142 we have $w_{3}=65, m_{3}=7, w_{2}=31, m_{2}=4, w_{1}=\emptyset$, $m_{1}=2$.

We now generate a Dyck path from $(2 n, 0)$ to $(0,0)$ (generate it backwards) using backward up-steps $((-1,1))$ and backward down-steps $((-1,-1))$.

Read $\pi$ from right to left. For each $m_{i}$ do $m_{i}-m_{i-1}$ up-steps (where we define $\left.m_{0}=0\right)$. For each $w_{i}$ do $\left|w_{i}\right|+1$ down-steps. So, for example, $\pi=6573142$ generates the Dyck path shown above.

We now state the main theorem of this section.

Theorem 3.2. Let $\pi \in S_{n}(123)$ and let $D_{\pi}$ be the associated Dyck path provided by Krattenthaler's bijection. Then $\pi$ is a backward derangement if and only if $D_{\pi}$ is hillfree.

Proof. We first demonstrate the "only-if" direction. Assume, for a contradiction, that $D_{\pi}$ has a hill. Notice that the hill cannot be at either end of the Dyck path for otherwise $\pi_{1}=n$ or $\pi_{n}=1$, both contradicting the fact that $\pi$ is a backward derangement. Hence, any hill must be an "interior" hill. Let $\pi=\pi(1) \pi(2)$ and $D_{\pi}=D_{\pi(1)} D_{\pi(2)}$ where $D_{\pi(1)}$ contains a hill on the right end and $D_{\pi(2)}$ contains no hill. Let $D_{\pi(2)}$ consist of $2 y$ steps. By construction, $\pi(2)$ contains the elements $1,2, \ldots, y$. Thus, in order for $D_{\pi(1)}$ to end with a hill, we must have $\pi_{n-y}(1)=y+1$, i.e., $\pi_{n+1-(y+1)}=y+1$, contradicting the fact that $\pi$ is a backward derangement.

We now give the "if" direction; we prove the contrapositive. Assume, for a contradiction, that $D_{\pi}$ has no hill. Let $x$ be the smallest integer such that $\pi_{n+1-x}=x$. Write $\pi=\pi(1) x \pi(2)$. From the above argument, we see that $\pi(2)$ cannot consist of the elements $1,2, \ldots, x-1$ only, for otherwise we would have a hill. Hence, there exists $y \in \pi(2)$ such that $y>x$. Consequently, there exists $w \in \pi(1)$ such that $w<x$. But then $w x y$ is a 123-pattern, a contradiction.

Coupling this theorem with Theorem 3.1, we immediately obtain the following corollary.

Corollary 3.3. For $n \geq 1, d_{n}(321)=F_{n}$, where $F_{n}$ is the $n^{\text {th }}$ Fine number. 
We can now investigate the relationship between $S_{n}^{k}(321)$ and similarity relations. To this end, we have the following theorem.

Theorem 3.4. For $n \geq 0, s_{n}^{k}(321)=\left|S R_{n}(k)\right|$.

Proof. We prove this by induction on $k$. The case $k=0$ is given by Corollary 3.3. Hence, we assume that there exists a bijection $\gamma_{n}^{k}: S_{n}^{k}(321) \rightarrow S R_{n}(k)$.

Let $\pi \in S_{n}^{k+1}(321)$ and let $f$ be the smallest fixed point. Write $\pi=\pi(1) f \pi(2)$ so that $\pi(2)$ contains $k$ fixed points. Note that since $\pi$ must be 321 -avoiding we must have $\pi(1) \in S_{f-1}^{0}(321)$ on the elements $1,2, \ldots, f-1$ and $\pi(2) \in S_{n-f}^{k}(321)$ on the elements $f+1, f+2, \ldots, n$. Let $\gamma_{f-1}^{0}(\pi(1))=t \in S R_{f}(0)$ and $\gamma_{n-f}^{k}(\pi(2))=r \in S R_{n-f}(k)$. Then define $\gamma_{n}^{k+1}(\pi)=t 0 r \in S R_{n}(k+1)$. To show this is a bijection, it is enough to give the inverse. This is obtained by noting that the position of the first 0 in the first occurrence of a double zero in an element of $S R_{n}(k+1)$ corresponds to the minimal fixed point.

\section{Weighted-Counting of 321-Avoiding Permutations}

In order to achieve one of our goals we must enumerate $S_{n}^{k}(321)$ via another approach. Before delving into our approach, we make the following definition.

Definition 4.1. Let $S$ be a finite set with each element $s \in S$ having a unique characteristic from $C=\left\{c_{1}, c_{2}, \ldots, c_{k}\right\}$, written as char $(s)$. The weight-enumerator of $S$ with respect to weight $(s)=x^{\text {char }(s)}$ is given by

$$
\sum_{i=1}^{k} s_{i} x^{c_{i}},
$$

where $s_{i}=\left|\left\{s \in S: \operatorname{char}(s)=c_{i}\right\}\right|$.

Applying this to our situation, let $\mathcal{A}_{n}=S_{n}(321)$ with $A_{n}(x)$ its weight-enumerator with respect to weight $(\pi)=x^{f(\pi)}$, where $f(\pi)$ is the number of fixed points of $\pi \in$ $S_{n}(321)$.

Recall that $\operatorname{Cat}(n)$ is the set of Catalan sequences, defined in Section 2. We will define a bijection $T: \mathcal{A}_{n} \rightarrow \operatorname{Cat}(n)$ as follows. If $n=1$, then $T(1)=1$. For $n \geq 2$ we define $c=T(\pi)$ recursively as follows.

Given a 321-avoiding permutation $\pi$ of length $n$, let $i$ be the place where $n$ is (i.e., $\left.\pi_{i}=n\right)$. If $\pi_{n}=n-1$ then let $\pi^{\prime}$ be $\pi_{1}, \ldots, \pi_{i-1}, n-1, \pi_{i+1}, \ldots, \pi_{n-1}$, otherwise let $\pi^{\prime}$ be $\pi$ with $n$ removed, i.e., $\pi^{\prime}=\pi_{1}, \ldots, \pi_{i-1}, \pi_{i+1}, \ldots, \pi_{n}$. Then $c=T(\pi)$ is defined to be $c^{\prime}=T\left(\pi^{\prime}\right)$ with $i$ appended at the end.

The inverse bijection $S: \operatorname{Cat}(n) \rightarrow \mathcal{A}_{n}$ is defined as follows. If $n=1$ then $S(1)=1$. If $n \geq 2$ and $c=c_{1} \cdots c_{n}$ is a Catalan sequence then $\pi=S(c)$ is defined recursively as follows. Let $c_{n}=i, c_{n-1}=j$, and let $c^{\prime}=c_{1} \cdots c_{n-2} c_{n-1}$ be $c$ with its last component removed. Let $\pi^{\prime}=S\left(c^{\prime}\right)$. If $i=j$ then let $\pi$ be the permutation obtained from $\pi^{\prime}$ by changing $n-1$ into $n$ and appending $n-1$ to the end, while if $j<i$ then let $\pi$ be the permutation obtained from $\pi^{\prime}$ by inserting $n$ at the $i^{\text {th }}$ place, i.e., $\pi=\pi_{1}^{\prime}, \ldots, \pi_{i-1}^{\prime}, n, \pi_{i}^{\prime}, \ldots, \pi_{n-1}^{\prime}$.

It is easy to prove, by induction on $n$, that $T S$ and $S T$ are identity mappings, and hence that $T$ is indeed a bijection. It is also easy to see that if $c_{1} \cdots c_{n}=T(\pi)$ then for 
$1 \leq i<n, \pi_{i}=i$ if and only if $c_{i}=i$ and $c_{i+1}=i+1$, and $\pi_{n}=n$ if and only if $c_{n}=n$. Hence $A_{n}(x)$ is equal to the weight-enumerator of Catalan sequences with respect to weight $(c)=x^{g(c)}$, where $g(c)$ is the number of $i$ 's such that $c_{i}=i$ and $c_{i+1}=i+1$ $(i<n)$ plus 1 if $c_{n}=n$.

Given a Catalan sequence $c$, let

$$
D(c)=\left\{i: 1 \leq i<n, c_{i}=i, c_{i+1}=i+1, \text { or } i=n \text { and } c_{n}=n\right\} .
$$

For example, $D(11345558)=\{3,4,8\}, D(11111111)=\emptyset$, and $D(112346)=\{6\}$. Note the weight of a Catalan sequence $c$ is $x^{|D(c)|}$.

In order to weight-enumerate the set of Catalan sequences it would be easier to use the inclusion-exclusion philosophy and consider the larger sets of marked Catalan sequences, which are the sets of pairs $(c, S)$ with $S \subset D(c)$ and weight defined by weight $(c, S)=(x-1)^{|S|}$. For example weight $(11345558,\{3,8\})=(x-1)^{2}$ and weight $(11345558,\{\})=(x-1)^{0}=1$. Since $x^{|D(c)|}=((x-1)+1)^{|D(c)|}=\sum_{S \subset D(c)}(x-$ $1)^{|S|}$, it follows that $A_{n}(x)$ is the weight-enumerator of marked Catalan sequences.

We now derive a recurrence. Given a marked Catalan sequence $\left(c_{1} \cdots c_{n}, S\right)$, if $1 \in S$ (i.e., 1 is marked) then we can get a smaller marked word by deleting $c_{1}=1$ and diminishing all indices and elements of $S$ by 1 . The weight-enumerator of this case is $(x-1) A_{n-1}(x)$.

If $1 \notin S$ (i.e., 1 is not marked), let $i$ be the smallest $i>1$ such that $c_{i}=i$, if it exists. Then $c_{2} \cdots c_{i-1}$ is a run-of-the-mill Catalan sequence of length $i-2$, while

$$
\left(c_{i}-(i-1) c_{i+1}-(i-1) \cdots c_{n}-(i-1), S-(i-1)\right)
$$

(by $S-(i-1)$ we mean the set $S$ with all of its elements reduced by $i-1)$ is a marked Catalan sequence of length $n-i+1$. The weight-enumerator of this case, for a given $i$, is $C_{i-2} A_{n-i+1}(x)(2 \leq i \leq n)$. Finally, if $c_{i}<i$ for all $2 \leq i \leq n$ then $c_{2} \cdots c_{n}$ is a typical Catalan sequence of length $n-1$. Hence we get

$$
A_{n}(x)=(x-1) A_{n-1}(x)+\left(\sum_{i=2}^{n} C_{i-2} A_{n-i+1}(x)\right)+C_{n-1},
$$

which can be rewritten as

$$
A_{n}(x)=(x-1) A_{n-1}(x)+\sum_{i=1}^{n} C_{i-1} A_{n-i}(x) .
$$

Introducing the generating function

$$
\phi(x, t)=\sum_{n=0}^{\infty} A_{n}(x) t^{n},
$$

and recalling the generating function for the Catalan numbers $\psi(t)=\frac{1-\sqrt{1-4 t}}{2 t}$, translates to

$$
\phi(x, t)=1+(x-1) t \phi(x, t)+t \phi(x, t) \psi(t) .
$$


Solving for $\phi(x, t)$ yields the explicit expression

$$
\phi(x, t)=\frac{2}{1-2(x-1) t+\sqrt{1-4 t}},
$$

which, in turn, implies (by multiplying the top and the bottom by $1-2(x-1) t-$ $\sqrt{1-4 t}$ ) that

$$
\phi(x, t)=\frac{1-x+\psi(t)}{2-x+t(x-1)^{2}} .
$$

To sum up we have the following theorem.

Theorem 4.1. Let $a_{n}(x)$ be the coefficient of $t^{n}$ in the Maclaurin expansion with respect to t of

$$
\frac{1-x+\psi(t)}{2-x+t(x-1)^{2}} \text {. }
$$

Then $A_{n}(x)=a_{n}(x)$.

We now investigate an interesting property of $a_{n}(x)$ that will be used in the next section. Expanding $a_{n}(x)$ in powers of $(x-1)$ and using (4.1), we may write

$$
a_{n}(x)=(x-1)^{n}+\sum_{i=0}^{n-1} \sum_{j=1}^{n-i} C_{j-1} a_{n-i-j}(x) \cdot(x-1)^{i} .
$$

Using (4.2) we prove the following crucial lemma.

Lemma 4.2. Let $a_{n}(x)=\sum_{k=0}^{n} b(n, k)(x-1)^{k}$ and define $b(x, y)=0$ if $y>x, x<0$, or $y<0$. Then, for $n \geq 1$ and $k \neq-1$, we have

$$
b(n, k)=b(n, k+1)+b(n-1, k-1)
$$

where $b(0,0)=1$.

Proof. Note that to render (4.3) valid when $n=k=0$, we would have to add a correction term of 1 to the right side, and to render (4.3) valid when $n \geq 0$ and $k=-1$ we would have to add $b(n, 0)=C_{n}$ to the left side.

We now prove (4.3) for $n \geq 1$ and $k \neq-1$ by induction on $n$. Since $a_{0}(x)=1$ and $a_{1}(x)=x$, the case $n=1$ is true. We now assume that $b(n-i, k)=b(n-i, k+1)+$ $b(n-i-1, k-1)$ for all $1 \leq i \leq n-1$ and $k \neq-1$.

To establish (4.3) for $n$ and all $k \neq-1$ we first note that this is clear if $k<-1$ or $k>n$. It is also clear for $k=n$ since $b(n, n)=b(n-1, n-1)=1$ by (4.2). To deal with $0 \leq k \leq n-1$ we use the fact that (4.2) yields

$$
b(n, k)=\sum_{i=0}^{n-1} \sum_{j=1}^{n-i} C_{j-1} b(n-i-j, k-i)
$$

for $0 \leq k \leq n-1$.

In particular, for $k=n-1$ this says that $b(n, n-1)=n$, so $b(n, n-1)=b(n, n)+$ $b(n-1, n-2)$ and (4.3) holds for $k=n-1$. 
For $0 \leq k \leq n-2$ we take (4.4) and apply (4.3) to each term on the right side, adding a correction term of $C_{n-k-1}$ when $i=k$ and $j=n-k$ (note that this case occurs since $k \neq-1$ ), and correction term $-\sum_{i=1}^{n-k-1} C_{i-1} C_{n-k-i-1}$ when $i=k+1$ (note that this case occurs since $k \neq n-1)$. Since the correction terms cancel, we have

$$
b(n, k)=\sum_{i=0}^{n-1} \sum_{j=1}^{n-i} C_{j-1} b(n-i-j, k-i+1)+\sum_{i=0}^{n-1} \sum_{j=1}^{n-i} C_{j-1} b(n-i-j-1, k-i-1) .
$$

Since $k+1 \leq n-1$, the first term on the right is $b(n, k+1)$ by (4.4). By (4.4) again, the second term on the right is $b(n-1, k-1)$ except for the terms in the double sum where $i=n-1$ or $j=n-i$. But these terms are all 0 because they involve values of $b(x, y)$ with $x<0$. This concludes the proof.

In [12], Strehl defines $a(n, k)$, for $n \geq 1$ and $1 \leq k \leq n$, as the number of similarity relations on $\{1,2, \ldots, n\}$ that have $k$ zeros. He proves that $a(n, k)=a(n, k+1)+a(n-$ $1, k-1)$ for $n \geq 2$, with $a(n, 1)=a(n, 2)=C_{n-1}$. It follows from Lemma 4.2 that $b(n, k)=a(n+1, k-1)$.

Using Lemma 4.2 we see that the beginning of the $b(n, k)$ table is as follows, where $n=0,1, \ldots$, corresponds to row $n$ and $k=0,1,2, \ldots$, is the $k^{\text {th }}$ term in from the left. This generates the so-called Catalan Triangle [11].

Table 1: Beginning of the Catalan triangle; values of $b(n, k)$.

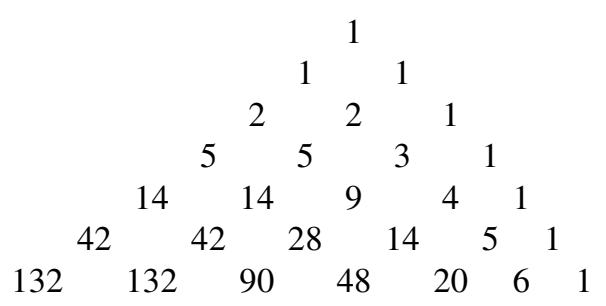

We also use Lemma 4.2 to prove the next lemma, which will be a crucial step in one of our main proofs.

Lemma 4.3. Let $a_{n}(x)=\sum_{k=0}^{n} b(n, k)(x-1)^{k}$. Then, for all $n, k \geq 1$, we have

$$
b(n, k)=\sum_{i=1}^{n} C_{i-1} b(n-i, k-1)
$$

where $b(n, 0)=C_{n}$, and $b(x, y)=0$ if $y>x$.

Proof. We use double induction; forward on $n$ and backward on $k$. We start with induction on $n$. From the table above, this clearly holds for $n=2$ and $1 \leq k \leq 2$. Hence, we assume that it holds for $n-1$ and $1 \leq k \leq n-1$ to show that it holds for $n$ and $1 \leq k \leq n$. We now perform backward induction on $k$. The base case $b(n, n)$ holds since $b(n, n)=b(n-1, n-1)=1$. Hence, we assume that the case $b(n, k+1)$ holds to show that the case $b(n, k)$ holds. 
We must do the case $k=1$ separately. This holds by the identity $C_{n}=\sum_{i=1}^{n} C_{i-1} C_{n-i}$ and the fact that $b(n, 1)=b(n, 0)=C_{n}$.

Now, from Lemma 4.2, we have $b(n, k)=b(n, k+1)+b(n-1, k-1)$ rendering the induction straightforward for $k \geq 2$.

\section{Weighted-Counting of 132-Avoiding Permutations}

Let $\mathcal{A}_{n}=S_{n}(321)$. Recall that $A_{n}(x)$ is its weight-enumerator with respect to the weight weight $(\pi)=x^{f(\pi)}$, where $f(\pi)$ is the number of fixed points of $\pi \in S_{n}(321)$. Let $\mathcal{B}_{n}=$ $S_{n}(132)$ and let $B_{n}(x)$ be its weight-enumerator with respect to the same weight. Our goal is to show that for $n \geq 0, A_{n}(x)=B_{n}(x)$.

We have that $\mathcal{B}_{n}$ is the set of 132 -avoiding bijections on $\{1,2, \ldots, n\}$. However, in order to weight-enumerate them, we must consider, more generally, the set $\mathcal{E}_{n, r}$ of bijections

$$
\pi:\{1,2, \ldots, n\} \rightarrow\{r+1, r+2, \ldots, r+n\}
$$

that avoid 132. Let $E_{n, r}(x)$ be the weight-enumerator of these bijections, with respect to the same weight. It is easy to see that $E_{n,-r}(x)=E_{n, r}(x)$, and that $E_{n, r}(x)=C_{n}$ if $r \geq n$, since in this situation no fixed point can occur. Of course $B_{n}(x)=E_{n, 0}(x)$.

We will now establish a recurrence for the $E_{n, r}(x)$. Consider $\pi \in \mathcal{E}_{n, r}$. Let $i$ be the location of $n+r$, i.e., the index $i$ for which $\pi_{i}=n+r$. Now the set of entries before the $i^{\text {th }}$ place must consist of the $i-1$ largest elements of the range, and the entries after the $i^{\text {th }}$ place must consist of the $n-i$ smallest elements, since otherwise a delinquent 132 will be formed. Hence, every member $\pi \in \mathcal{E}_{n, r}$ gives rise to a pair $\left(\pi^{\prime}, \pi^{\prime \prime}\right)$ where $\pi^{\prime} \in \mathcal{E}_{i-1, n-i+r}$ and $\pi^{\prime \prime} \in \mathcal{E}_{n-i, r-i}$. Furthermore, the weight of $\pi$ is the product of the weights of $\pi^{\prime}$ and $\pi^{\prime \prime}$, except when $r=0$ and $i=n$ in which case we removed a fixed point, and we have an extra factor of $x$. Let $I_{S}$ be the characteristic function, i.e., $I_{S}=1$ if $S$ is true, and 0 otherwise. The above argument gives the following non-linear recurrence.

$$
E_{n, r}(x)=\sum_{i=1}^{n} E_{i-1, n-i+r}(x) E_{n-i, r-i}(x)+(x-1) E_{n-1,0}(x) I_{\{r=0\}} .
$$

Changing $E_{n-i, r-i}(x)$ to $E_{n-i, i-r}(x)$ when $i>r$ and using the fact that $E_{i-1, n-i+r}(x)=$ $C_{i-1}$ when $n-i+r \geq i-1$, i.e., $i \leq\left\lfloor\frac{n+r+1}{2}\right\rfloor$, and $E_{n-i, i-r}(x)=C_{n-i}$ when $i-r \geq n-i$, i.e., $i \geq\left\lceil\frac{n+r}{2}\right\rceil$, we get a simplified recurrence, which is linear in $E_{n, r}$ :

$$
\begin{aligned}
E_{n, r}(x)= & \sum_{i=1}^{r} C_{i-1} E_{n-i, r-i}(x)+\sum_{i=r+1}^{\left\lfloor\frac{n+r+1}{2}\right\rfloor} C_{i-1} E_{n-i, i-r}(x) \\
& +\sum_{i=\left\lfloor\frac{n+r+1}{2}\right\rfloor+1}^{n} E_{i-1, n-i+r}(x) C_{n-i}+(x-1) E_{n-1,0}(x) I_{\{r=0\}} .
\end{aligned}
$$

We are now able to prove the following theorem. 
Theorem 5.1. Let $a_{n}(x)$ be the weight-enumerator of 321-avoiding permutations with respect to weight $(\pi)=x^{f(\pi)}$, where $f(\pi)$ is the number of fixed points in $\pi \in S_{n}(321)$. Let $E_{n, r}(x)$ be the weight-enumerator of 132-avoiding bijections from $\{1,2, \ldots, n\}$ to $\{r+1, r+2, \ldots, r+n\}$ with respect to the same weight. Then, for $r \geq 0$, we have

$$
E_{n, r}(x)=a_{n}(x)+(1-x) \sum_{i=1}^{r} C_{i-1} a_{n-i}(x),
$$

where we define $a_{j}(x)=0$ for $j<0$.

Proof. Denote by $e_{n, r}(x)$ the right side of (5.1). We must show for all $n$ (keeping $r$ as a parameter) that

$$
\begin{aligned}
e_{n, r}(x)= & \sum_{i=1}^{r} C_{i-1} e_{n-i, r-i}(x)+\sum_{i=r+1}^{\left\lfloor\frac{n+r+1}{2}\right\rfloor} C_{i-1} e_{n-i, i-r}(x) \\
& +\sum_{i=\left\lfloor\frac{n+r+1}{2}\right\rfloor+1}^{n} e_{i-1, n-i+r}(x) C_{n-i}+(x-1) e_{n-1,0}(x) I_{\{r=0\}} .
\end{aligned}
$$

In other words, we have to prove

$$
\begin{aligned}
& a_{n}(x)+(1-x) \sum_{i=1}^{r} C_{i-1} a_{n-i}(x) \\
& =\sum_{i=1}^{r} C_{i-1}\left(a_{n-i}(x)+(1-x) \sum_{j=1}^{r-i} C_{j-1} a_{n-i-j}(x)\right) \\
& \quad+\sum_{i=r+1}^{\left.\frac{n+r+1}{2}\right\rfloor} C_{i-1}\left(a_{n-i}(x)+(1-x) \sum_{j=1}^{i-r} C_{j-1} a_{n-i-j}(x)\right) \\
& \quad+\sum_{i=\left\lfloor\frac{n+r+1}{2}\right\rfloor+1}^{n} C_{n-i}\left(a_{i-1}(x)+(1-x) \sum_{j=1}^{n-i+r} C_{j-1} a_{i-j-1}(x)\right) \\
& \quad+(x-1) a_{n-1}(x) I_{\{r=0\}},
\end{aligned}
$$

which has been checked by the Maple package AARON for $n \leq 50$. AARON was written by the third author (with additions by the first author) and is available at each of these author's website (http://math.colgate.edu/ aaron and http://www.math. rutgers. edu/ zeilberg).

Let the left side of (5.2) be $l_{n}(x)$ and the right side be $r_{n}(x)$. Write

$$
l_{n}(x)=\sum_{k=0}^{n} s(n, k)(x-1)^{k} \text { and } r_{n}(x)=\sum_{k=0}^{n} t(n, k)(x-1)^{k} .
$$

If we can show that for any $n, s(n, k)=t(n, k)$ for all $0 \leq k \leq n$, then we will be done.

We must take care of the case $k=0$ separately. This case holds since $b(n, 0)=C_{n}$ and $C_{n}=\sum_{i=1}^{n} C_{i-1} C_{n-i}$. 
We now use double induction on $n \geq 2$ and $k \geq 0$; forward induction on $n$ and backward induction on $k$.

We start by inducting on $n$. Since Maple has given us the base case, i.e., $s(2, k)=$ $t(2, k)$ for $0 \leq k \leq 2$, we may assume that $s(n-1, k)=t(n-1, k)$ for all $0 \leq k \leq n-1$. We must show that $s(n, k)=t(n, k)$ for all $0 \leq k \leq n$.

We proceed via backward induction on $k$. For our base case we must show that $s(n, n)=t(n, n)$. Gathering the $(x-1)^{n}$ terms in (5.2) we have $(x-1)^{n}-(x-1)^{n} I_{\{r \neq 0\}}$ on the left side of (5.2), and $(x-1)^{n} I_{\{r=0\}}$ on the right side of (5.2). Since $(x-1)^{n}=$ $(x-1)^{n} I_{\{r \neq 0\}}+(x-1)^{n} I_{\{r=0\}}$, we have $s(n, n)=t(n, n)$

We now assume that $s(n, k+1)=t(n, k+1)$ to show that $s(n, k)=t(n, k)$ for $k \geq 1$. Letting $a_{n}(x)=\sum_{k=0}^{n} b(n, k)(x-1)^{k}$, with $b(0,0)=1$ and $b(x, y)=0$ if $y>x, x<0$, or $y<0$, we must show that

$$
\begin{aligned}
& b(n, k)-\sum_{i=1}^{r} C_{i-1} b(n-i, k-1) \\
& =\sum_{i=1}^{r} C_{i-1}\left(b(n-i, k)-\sum_{j=1}^{r-i} C_{j-1} b(n-i-j, k-1)\right) \\
& \quad+\sum_{i=r+1}^{\left\lfloor\frac{n+r+1}{2}\right\rfloor} C_{i-1}\left(b(n-i, k)-\sum_{j=1}^{i-r} C_{j-1} b(n-i-j, k-1)\right) \\
& +\sum_{i=\left\lfloor\frac{n+r+1}{2}\right\rfloor+1}^{n} C_{n-i}\left(b(i-1, k)-\sum_{j=1}^{n-i+r} C_{j-1} b(i-j-1, k-1)\right) \\
& +b(n-1, k-1) I_{\{r=0\}} .
\end{aligned}
$$

The cases $r \geq n$ are straightforward and are left to the reader. Below, we assume that $r<n$.

Using Lemma 4.2, and the inductive hypothesis for $b(n, k+1)$ we must show that

$$
\begin{aligned}
& b(n-1, k-1)-\sum_{i=1}^{r} C_{i-1} b(n-i-1, k-2) \\
& =\sum_{i=1}^{r} C_{i-1} b(n-i-1, k-1)-\sum_{i=1}^{r} \sum_{j=1}^{r-i} C_{i-1} C_{j-1} b(n-i-j-1, k-2) \\
& \quad+\sum_{i=r+1}^{\left\lfloor\frac{n+r+1}{2}\right\rfloor} C_{i-1} b(n-i-1, k-1)-\sum_{i=r+1}^{\left\lfloor\frac{n+r+1}{2}\right\rfloor} \sum_{j=1}^{i-r} C_{i-1} C_{j-1} b(n-i-j-1, k-2) \\
& \quad+\sum_{i=\left\lfloor\frac{n+r+1}{2}\right\rfloor+1}^{n} C_{n-i} b(i-2, k-1)-\sum_{i=\left\lfloor\frac{n+r+1}{2}\right\rfloor+1}^{n} \sum_{j=1}^{n-i+r} C_{n-i} C_{j-1} b(i-j-2, k-2) \\
& \quad-C_{\left\lfloor\frac{n+r+1}{2}\right\rfloor-1} C_{n-\left\lfloor\frac{n+r+1}{2}\right\rfloor-1} I_{\{k=1\}}+b(n-2, k-2) I_{\{r=0\}},
\end{aligned}
$$


where the second to last term occurs for $i=\left\lfloor\frac{n+r+1}{2}\right\rfloor$ and $j=n-i$ in the second double sum of (5.3) (as this gives $b(0,0)$ which is equal to 1 and not 0 as would be given by the recurrence in Lemma 4.2).

We must consider two cases: $n+r$ even and $n+r$ odd. Assume that $n+r$ is even; the case where $n+r$ is odd is similar. For $n+r$ even we have $\left\lfloor\frac{n+r+1}{2}\right\rfloor=\left\lfloor\frac{n+r}{2}\right\rfloor$. Using the inductive hypothesis for $b(n-1, k-1)$ we are reduced to showing that

$$
C_{n-\left\lfloor\frac{n+r}{2}\right\rfloor-1} b\left(\left\lfloor\frac{n+r}{2}\right\rfloor-1, k-1\right)=C_{\left\lfloor\frac{n+r}{2}\right\rfloor-1} C_{n-\left\lfloor\frac{n+r}{2}\right\rfloor-1}
$$

if $k=1$, which holds since $b(n, 0)=C_{n}$, or

$C_{n-\left\lfloor\frac{n+r}{2}\right\rfloor-1} b\left(\left\lfloor\frac{n+r}{2}\right\rfloor-1, k-1\right)=C_{n-\left\lfloor\frac{n+r}{2}\right\rfloor-1} \sum_{i=1}^{\left\lfloor\frac{n+r}{2}\right\rfloor-1} C_{i-1} b\left(\left\lfloor\frac{n+r}{2}\right\rfloor-i-1, k-2\right)$

if $k \geq 2$, which holds by Lemma 4.3 , thereby completing the proof.

\section{D-Wilf Classes and h-Wilf Classes}

No discussion of restricted permutations is complete without the discussion of Wilf classes, defined below, where $S_{n}(T), T \subseteq S_{m}$, is the set of $\pi \in S_{n}$ which avoid all patterns in $T$ and where $s_{n}(T)=\left|S_{n}(T)\right|$.

Definition 6.1. Let $S_{1}, S_{2} \subseteq S_{m}$. If $s_{n}\left(S_{1}\right)=s_{n}\left(S_{2}\right)$ for all $n \geq m$ then we say that $S_{1}$ and $S_{2}$ are in the same Wilf class, or are Wilf equivalent.

Since we have refined the investigation of restricted permutations, we refine the notion of Wilf class with the following definition, where $S_{n}^{k}(T), T \subseteq S_{m}$, is the set of $\pi \in S_{n}$ with exactly $k$ fixed points which avoid all patterns in $T$ and where $s_{n}^{k}(T)=$ $\left|S_{n}^{k}(T)\right|$. We use $D_{n}(T)$ and $d_{n}(T)$ to represent $S_{n}^{0}(T)$ and $s_{n}^{0}(T)$, respectively.

Definition 6.2. Let $S_{1}, S_{2} \subseteq S_{m}$. If $d_{n}\left(S_{1}\right)=d_{n}\left(S_{2}\right)$ for all $n \geq m$ we say that $S_{1}$ and $S_{2}$ are in the same D-Wilf class, or are D-Wilf equivalent. For $h>0$, if $s_{n}^{h}\left(S_{1}\right)=s_{n}^{h}\left(S_{2}\right)$ for all $n \geq m, h$, we say that $S_{1}$ and $S_{2}$ are in the same $h$-Wilf class, or are $h$-Wilf equivalent.

We will have need of the following lemma in the proofs below.

Lemma 6.1. Let $\gamma \in S_{n}$ be given by $\gamma_{i}=n+1-i$ for $1 \leq i \leq n$. For $\pi \in S_{n}$, let $\pi^{\star}=$ $\gamma \pi \gamma^{-1}$. Then, for all $\pi, \pi$ and $\pi^{\star}$ have the same number of fixed points. Furthermore, the number of occurrences of the pattern 213 (respectively 312) in $\pi$ equals the number of occurrences of the pattern 132 (respectively 231) in $\pi^{\star}$.

Proof. Since $\pi^{\star}$ is obtained from $\pi$ by conjugation, $\pi$ and $\pi^{\star}$ have the same number of fixed points. If $i<j<k$ are such that $\pi_{i} \pi_{j} \pi_{k}$ is an occurrence of 213 (resp. 312), then $\gamma_{k}<\gamma_{j}<\gamma_{i}$ are such that $\pi_{\gamma_{k}}^{\star} \pi_{\gamma_{j}}^{\star} \pi_{\gamma_{i}}^{\star}$ is an occurrence of 132 (resp. 231).

We now state some results about refined Wilf classes.

Theorem 6.2. There are exactly three D-Wilf classes of patterns of length 3. 
Proof. Applying Theorem 5.1 with $r=0$ we see that, in particular, $d_{n}(321)=d_{n}(132)$. Lemma 6.1 gives us $d_{n}(132)=d_{n}(213)$ and $d_{n}(231)=d_{n}(312)$.

Lastly, we note that $d_{4}(123)=7, d_{4}(132)=6$, and $d_{4}(231)=4$, thereby giving three $D$-Wilf classes.

Theorem 6.3. For any $h>0$, there are exactly three $h$-Wilf classes of patterns of length 3.

Proof. We already have $s_{n}^{h}(132)=s_{n}^{h}(213)=s_{n}^{h}(321)$ and $s_{n}^{h}(231)=s_{n}^{h}(312)$ for all $1 \leq h \leq n$. We now show that for $h>0,\left\{s_{n}^{h}(132)\right\}_{n \geq 3},\left\{s_{n}^{h}(231)\right\}_{n \geq 3}$, and $\left\{s_{n}^{h}(123)\right\}_{n \geq 3}$ are different sequences, thereby showing that there are three $h$-Wilf classes for $h>0$.

First, since $s_{5}^{1}(123)=20, s_{5}^{1}(231)=16, s_{5}^{1}(132)=13, s_{5}^{2}(123)=2, s_{5}^{2}(231)=8$, and $s_{5}^{2}(132)=6$ we have exactly three $h$-Wilf classes for $h=1,2$. Next, note that for $h \geq 3, s_{n}^{h}(123)=0$ since with three fixed points we have a 123 pattern. We conclude by showing that $s_{h+2}^{h}(132)=h+1$ and $s_{h+2}^{h}(312) \geq 2 h+1$, thereby giving exactly three $h$-Wilf classes for all $h \geq 3$.

Let $\pi \in S_{h+2}^{h}(132)$. To show that $s_{h+2}^{h}(132)=h+1$, note that we can only have two entries of $\pi$ which are not fixed points. Furthermore, 1 cannot be a fixed point, for otherwise all other entries must be fixed in order to avoid the 132 pattern. Hence, we have the freedom to pick exactly one of $2,3 \ldots, h+2$ to be a non-fixed point. This gives $h+1$ choices.

Next, we show that $s_{h+2}^{h}(312) \geq s_{h+1}^{h-1}(312)+2$, which gives $s_{h+2}^{h}(312) \geq 2 h+1$ since $s_{3}^{1}(312)=3$. Consider the following procedure. Let $\pi \in S_{h+1}^{h-1}(312)$. Let $\widehat{\pi}_{i}=\pi_{i}+$ 1 for $1 \leq i \leq h+1$. Construct $X=\left\{1 \widehat{\pi}_{1} \widehat{\pi}_{2} \cdots \widehat{\pi}_{h+1}\right\} \cup\left\{\pi_{1} \pi_{2} \cdots \pi_{h+1}(h+2): \pi_{1} \neq 1\right\}$. It is clear that these sets are disjoint and that $X \subseteq S_{h+2}^{h}(312)$. All that remains to be shown is that $\left|\left\{\pi_{1} \pi_{2} \cdots \pi_{h+1}(h+2): \pi_{1} \neq 1\right\}\right|=2$. To see this, note that $S_{3}^{1}(312)=$ $\{132,321,213\}$ contains two elements with $\pi_{1} \neq 1$. By construction of the above procedure, these two elements beget two elements in $S_{4}^{2}(312)$ such that $\pi_{1} \neq 1$. This concludes the proof.

We may further show, using Lemma 6.1, another result concerning Wilf classes. First, we remind the reader of the following definition from [6].

Definition 6.3. Let $S \subseteq S_{m}$ and let $T$ be a multiset of $S_{m}$. Define $S_{n}(S ; T)$ to be the set of $\pi \in S_{n}$ which avoid all patterns in $S$ and contain each element, including multiplicities, in $T$ exactly once. Let $s_{n}(S ; T)=\left|S_{n}(S ; T)\right|$. Let $S_{1}, S_{2} \subset S_{m}$ and let $T_{1}, T_{2}$ be multisets of $S_{m}$. If $s_{n}\left(S_{1} ; T_{1}\right)=s_{n}\left(S_{2} ; T_{2}\right)$ for all $n \geq m$ we say that $\left(S_{1} ; T_{1}\right)$ and $\left(S_{2} ; T_{2}\right)$ are in the same almost-Wilf class, or are almost-Wilf equivalent. We drop the set notation for a singleton set.

Next, we refine this definition.

Definition 6.4. Define $S_{n}^{k}(S ; T)$ to be those permutations in $S_{n}(S ; T)$ with exactly $k$ fixed points. Let $s_{n}^{k}(S ; T)=\left|S_{n}^{k}(S ; T)\right|$. When $k=0$ we write $D_{n}(S ; T)$, and $d_{n}(S ; T)$, respectively. If $d_{n}\left(S_{1} ; T_{1}\right)=d_{n}\left(S_{2} ; T_{2}\right)$ for all $n \geq m$ we say that $\left(S_{1} ; T_{1}\right)$ and $\left(S_{2} ; T_{2}\right)$ are in the same almost-D-Wilf class, or are almost-D-Wilf equivalent. For $h>0$, if $s_{n}^{h}\left(S_{1} ; T_{1}\right)=s_{n}^{h}\left(S_{2} ; T_{2}\right)$ for all $n \geq m, h$, we say that $\left(S_{1} ; T_{1}\right)$ and $\left(S_{2} ; T_{2}\right)$ are in the same almost-h-Wilf class, or are almost-h-Wilf equivalent. 
Theorem 6.4. Consider $D_{n}(\emptyset, \alpha), \alpha \in S_{3}$. For these permutations there are exactly four almost-D-Wilf classes.

Proof. We first prove that $d_{n}(0 ; 321)=0$ for all $n \geq 3$. Let $c b a$ be a 321 pattern in $\pi \in S_{n}$. Write $\pi=W c X b Y a Z$. In order to avoid another 321 pattern we see that for $w \in W$ and $x \in X$ we must have $w, x<b$. Furthermore, for $y \in Y$ and $z \in Z$ we must have $y, z>b$. Hence, $b$ is a fixed point. Thus, the restriction of having exactly one 321 pattern implies a fixed point must be present.

Using Lemma 6.1, we see that $d_{n}(\emptyset ; 132)=d_{n}(\emptyset ; 213)$ and $d_{n}(\emptyset ; 231)=d_{n}(\emptyset ; 312)$. Lastly, since $d_{5}(\emptyset ; 123)=14, d_{5}(\emptyset ; 132)=8$, and $d_{5}(\emptyset ; 231)=6$, we have exactly four almost- $D$-Wilf classes.

\section{Enumeration and Other Results}

We start this section by tabulating $s_{n}^{k}(\alpha)$ for $n \leq 8$ and $0 \leq k \leq 8$ for all $\alpha \in S_{3}$ using the fact that there are only three $D$-Wilf classes.

Table 2: Values of $s_{n}^{k}(132)=s_{n}^{k}(321)=s_{n}^{k}(213)$.

\begin{tabular}{l|ccccccccc}
$n \backslash{ }^{k}$ & 0 & 1 & 2 & 3 & 4 & 5 & 6 & 7 & 8 \\
\hline 0 & 1 & & & & & & & & \\
1 & 0 & 1 & & & & & & & \\
2 & 1 & 0 & 1 & & & & & & \\
3 & 2 & 2 & 0 & 1 & & & & & \\
4 & 6 & 4 & 3 & 0 & 1 & & & & \\
5 & 18 & 13 & 6 & 4 & 0 & 1 & & & \\
6 & 57 & 40 & 21 & 8 & 5 & 0 & 1 & & \\
7 & 186 & 130 & 66 & 30 & 10 & 6 & 0 & 1 \\
8 & 622 & 432 & 220 & 96 & 40 & 12 & 7 & 0 & 1
\end{tabular}

Table 3: Values of $s_{n}^{k}(231)=s_{n}^{k}(312)$.

\begin{tabular}{|c|c|c|c|c|c|c|c|}
\hline$n \backslash k$ & 0 & 1 & 2 & 3 & & & $\begin{array}{lll}6 & 78\end{array}$ \\
\hline$\overline{0}$ & 1 & & & & & & \\
\hline 1 & 0 & 1 & & & & & \\
\hline 2 & 1 & 0 & 1 & & & & \\
\hline 3 & 1 & 3 & 0 & 1 & & & \\
\hline 4 & 4 & 4 & 5 & 0 & 1 & & \\
\hline 5 & 10 & 16 & 8 & 7 & 0 & 1 & \\
\hline 6 & 31 & 44 & 35 & 12 & 9 & & 1 \\
\hline t & 94 & 146 & 102 & 59 & & & \\
\hline 8 & 303 & 464 & 362 & 180 & 872 & 201 & 13 \\
\hline
\end{tabular}


Table 4: Values of $s_{n}^{k}(123)$.

\begin{tabular}{l|ccccccccc}
$n \backslash k$ & 0 & 1 & 2 & 3 & 4 & 5 & 6 & 7 & 8 \\
\hline 0 & 1 & & & & & & & & \\
1 & 0 & 1 & & & & & & \\
2 & 1 & 0 & 1 & & & & & & \\
3 & 2 & 3 & 0 & 0 & & & & \\
4 & 7 & 4 & 3 & 0 & 0 & & & & \\
5 & 20 & 20 & 2 & 0 & 0 & 0 & & & \\
6 & 66 & 48 & 18 & 0 & 0 & 0 & 0 & & \\
7 & 218 & 183 & 28 & 0 & 0 & 0 & 0 & 0 & \\
8 & 725 & 552 & 153 & 0 & 0 & 0 & 0 & 0 & 0
\end{tabular}

From Corollary 3.3, Theorem 5.1, and Lemma 6.1 we have $d_{n}(132)=d_{n}(321)=$ $d_{n}(213)=F_{n}$ for $n \geq 1$, where $F_{n}$ is the $n^{\text {th }}$ Fine number.

From the above triangles it appears that $d_{n}(231)<F_{n}$ and $d_{n}(123)>F_{n}$ for $n \geq 3$. Unfortunately, we were unable to prove the latter assertion. However, we can prove the former via a bijection similar to the one found in [4].

Theorem 7.1. Let $F_{n}$ be the $n^{\text {th }}$ Fine number. For all $n \geq 3$, we have $d_{n}(231)<F_{n}$.

Proof. We first consider the following bijection $\gamma: S_{n}(132) \rightarrow S R_{n}$. Let $\pi \in S_{n}(132)$ and $\pi=\pi_{1} \pi_{2} \cdots \pi_{n}$. Let $s=s_{n} s_{n-1} \cdots s_{1}=\gamma(\pi)$ where $s_{i}$ is the number of entries in $\pi_{i+1} \pi_{i+2} \cdots \pi_{n}$ which are larger than $\pi_{i}$. For example, $\gamma(456312)=010012$.

The fact that $\pi$ is 132 -avoiding guarantees that $s_{i-1} \leq s_{i}+1$. Hence, $s \in S R_{n}$. The inverse bijection is obvious.

We now prove that if $s \notin S R_{n}(0)$ then $\gamma^{-1}(s)$ is not a backward derangement. By observing that if $\pi \in S_{n}(132)$ is a backward derangement then when $\pi$ is read from right to left it becomes a member of $D_{n}(231)$, we can conclude that $D_{n}(231) \leq\left|S R_{n}(0)\right|=F_{n}$.

We must first take care of the case where $\gamma(\pi)$ produces $s_{1}=0$. In this case, it is obvious that $\pi_{1}=n$ and hence $\pi$ is not a backward derangement. We now assume that the first case of a double zero starts with $s_{i+1}, i \neq 0$, so that $s_{i+1}=s_{i}=0$. The bijection implies that $\pi_{i}>\pi_{i+1}$. If there exists $\pi_{k}, k<i$, with $\pi_{k}<\pi_{i+1}$ then $\pi_{k} \pi_{i+1} \pi_{i}$ is an occurrence of the pattern 132, and hence is not allowed. Thus, all elements less than $\pi_{i+1}$ are to the right of $\pi_{i+1}$. This implies that $\pi_{n+1-\pi_{i+1}}=\pi_{i+1}$.

We must now prove that the inequality is strict. We do this by giving $\pi \in S_{n}(132)$ which is not a backward derangement for which $\gamma(\pi)$ is nonsingular. For $n$ odd we have $\gamma(123 \cdots n)=012 \cdots n-1$ and for $n$ even $\gamma(234 \cdots(n-1) 1 n)=01123 \cdots n-2$. Noting that both of the similarity relations produced by $\gamma$ are nonsingular and correspond to permutations of $S_{n}(132)$ which are not backward derangements completes the proof.

We now turn our attention to the patterns 132, 321, and 213 (all of which are in the same $D$-Wilf class). Using Theorem 5.1, we are able to derive some formulas (recursive and "closed" form) for $s_{n}^{k}(\alpha), \alpha \in\{132,321,213\}$. First, we rederive items 2 and 4 of Theorem 2.1. 
Let $r=n$ in Theorem 5.1. Since $E_{n, n}=C_{n}$ we have, using (4.1),

$$
C_{n}=2 a_{n}(x)-x a_{n}(x)+(1-x)^{2} a_{n-1}(x) .
$$

Equating the constant terms gives us $C_{n}=2 d_{n}(\alpha)+d_{n-1}(\alpha)$ for $\alpha \in\{132,321,213\}$. Hence, $C_{n}=2 F_{n}+F_{n-1}$ is rederived. From here the derivation of item 4 in Theorem 2.1 uses either a straightforward induction or telescoping sum.

We can, of course, use (7.1) to derive recurrences for $s_{n}^{k}(\alpha), \alpha \in\{132,321,213\}$, for $k \neq 0$. To this end, we have the following.

Theorem 7.2. For $0 \leq k \leq n$, let $F_{n}^{k}=s_{n}^{k}(\alpha)$, $\alpha \in\{132,321,213\}$. Define $F_{n}^{-1}=0$. For $n \geq 2$ and $1 \leq k \leq n$, we have

$$
2 F_{n}^{k}+F_{n-1}^{k}=F_{n}^{k-1}+2 F_{n-1}^{k-1}-F_{n-1}^{k-2} .
$$

Proof. Equate the coefficients of $x^{k}$ in (7.1).

We can now use a telescoping sum to show the following.

Theorem 7.3. Let $C_{n}$ and $F_{n}$ be the $n^{\text {th }}$ Catalan and Fine number, respectively. Let $\alpha \in\{132,321,213\}$. For $n \geq 1$, we have

$$
s_{n}^{1}(\alpha)=\frac{1}{4} \sum_{i=0}^{n-1}\left(\frac{-1}{2}\right)^{i}\left(C_{n-i}+3 F_{n-i-1}\right) .
$$

Proof. Routine.

Other formulas for $s_{n}^{k}(\alpha), \alpha \in\{132,321,213\}, k \geq 2$, similar to the one in Theorem 7.3 (which are based on Catalan and Fine numbers) can be derived using Theorem 7.2. However, these formulas are rather cumbersome. Instead, we present some nicer formulas. First, we have the following lemma, which introduces a generalization of the Catalan numbers.

Lemma 7.4. Let $n \geq 1$. Write $a_{n}(x)=\sum_{k=0}^{n} b(n, k)(x-1)^{k}$. Then

$$
b(n, k)=\frac{k+1}{n+1}\left(\begin{array}{c}
2 n-k \\
n
\end{array}\right)
$$

Proof. Using Lemma 4.2 it is routine to verify the stated formula.

Since Lemma 7.4 gives us a natural generalization of the Catalan numbers, we define

$$
C_{n}^{(k)}=\frac{k+1}{n+1}\left(\begin{array}{c}
2 n-k \\
n
\end{array}\right) .
$$

Using Lemma 7.4 it is easy to prove the following formulas.

Theorem 7.5. Let $\alpha \in\{132,321,213\}$. For $n \geq 1,0 \leq k \leq n$, we have 


$$
s_{n}^{k}(\alpha)=\sum_{j=0}^{n-k}(-1)^{j}\left(\begin{array}{c}
j+k \\
k
\end{array}\right) C_{n}^{(k+j)},
$$

i.e.,

$$
s_{n}^{k}(\alpha)=\sum_{j=0}^{n-k}(-1)^{j} \frac{j+k+1}{n+1}\left(\begin{array}{c}
2 n-k-j \\
n
\end{array}\right)\left(\begin{array}{c}
j+k \\
k
\end{array}\right) .
$$

Proof. We have $a_{n}(x)=\sum_{j=0}^{n} C_{n}^{(j)}(x-1)^{j}$. Expanding $(x-1)^{j}$ we get

$$
a_{n}(x)=\sum_{j=0}^{n} \sum_{i=0}^{j}(-1)^{j-i} C_{n}^{(j)}\left(\begin{array}{l}
j \\
i
\end{array}\right) x^{i} .
$$

Equating the coefficients of $x^{k}$, we get

$$
s_{n}^{k}(\alpha)=\sum_{j=k}^{n}(-1)^{j-k} C_{n}^{(j)}\left(\begin{array}{l}
j \\
k
\end{array}\right),
$$

which, after a change of variable, gives the desired result.

We now harvest some other interesting results.

From Theorem 7.5 we have the following corollary which relates $C_{n}^{(k)}$ and $C_{n}$.

Corollary 7.6. Let $C_{n}$ be the $n^{\text {th }}$ Catalan number and let $C_{n}^{(k)}$ be as defined in (7.2). Then, for $n \geq 0$, we have

$$
C_{n}=\sum_{k=0}^{n} \sum_{j=0}^{n-k}(-1)^{j}\left(\begin{array}{c}
j+k \\
k
\end{array}\right) C_{n}^{(k+j)} .
$$

Proof. Since $\sum_{k=0}^{n} s_{n}^{k}(\alpha)=C_{n}$ for any $\alpha \in S_{3}$, the result is immediate.

We can also use Theorem 7.5 to rederive a formula for the Fine numbers given in [1].

Corollary 7.7. Let $F_{n}$ be the $n^{\text {th }}$ Fine number. For $n \geq 1$, we have

$$
F_{n}=\sum_{j=0}^{n}(-1)^{j} C_{n}^{(j)}
$$

As a consequence of $C_{n}=2 F_{n}+F_{n-1}$ and $F_{n}=d_{n}(\alpha)$ for $\alpha \in\{132,213,321\}$ we get the following theorem.

Theorem 7.8. Let $\alpha \in\{132,213,321\}$ and let $F(\pi)$ be the set of fixed points of $\pi \in$ $S_{n}(\alpha)$. Define

$$
T_{n}(\alpha)=\left\{\pi \in S_{n}(\alpha): F(\pi) \cap\{1,2, \ldots, n-1\} \neq \emptyset\right\} .
$$

Then $\left|T_{n}(\alpha)\right|=F_{n}$, where $F_{n}$ is the $n^{\text {th }}$ Fine number.

Proof. We have $C_{n}=s_{n}(\alpha)$ and $F_{n}=d_{n}(\alpha)$. If $U_{n}(\alpha)=\left\{\pi_{1} \cdots \pi_{n-1} n: \pi \in D_{n-1}(\alpha)\right\}$ then $S_{n}(\alpha)$ is the disjoint union $S_{n}(\alpha)=D_{n}(\alpha) \cup T_{n}(\alpha) \cup U_{n}(\alpha)$. Since $C_{n}=2 F_{n}+F_{n-1}$, the result follows. 


\section{References}

1. E. Deutsch, Dyck path enumeration, Discrete Math. 204 (1999) 167-202.

2. T. Fine, Extrapolation when very little is known, Inform. and Control 16 (1970) 331-359.

3. D. Knuth, The Art of Computer Programming, Vol. 3, Addison-Wesley, Reading, MA, 1973.

4. C. Krattenthaler, Permutations with restricted patterns and Dyck paths, Adv. Appl. Math. 27 (2001) 510-530.

5. D. Richards, Ballot sequences and restricted permutations, Ars Combin. 25 (1988) 83-86.

6. A. Robertson, Permutations restricted by two distinct patterns of length three, Adv. Appl. Math. 27 (2001) 548-561.

7. D.G. Rogers, Similarity relations on finite ordered sets, J. Combin. Theory, Ser. A 23 (1977) 88-98.

8. F. Schmidt and R. Simion, Card shuffling and a transformation in $S_{n}$, Aeq. Math. 44 (1992) $11-34$.

9. L.W. Shapiro, A Catalan triangle, Discrete Math. 14 (1976) 83-90.

10. R. Simion and F. Schmidt, Restricted permutations, Europ. J. Combin. 6 (1985) 383-406.

11. N.J.A. Sloane, The on-line encyclopedia of integer sequences, http://www.research.att.com/ njas/sequences, A009766.

12. V. Strehl, A Note on similarity relations, Discrete Math. 19 (1977) 99-101.

13. J. West, Permutations with forbidden subsequences and stack sortable permutations, Ph.D. Thesis, MIT, 1990. 